\title{
Maxillofacial Radiology 187
}

SADJ February 2021, Vol. 76 No. 1 p41

\section{CJ Nortjé}

Below are six normal anatomical landmarks (ABC), (DEF), (GH), (I), $(\mathrm{J})$ and $(\mathrm{K})$ which could due to faulty angulation or lack of knowledge be diagnosed as undelying pathology or traumatic injury. Identify the anatomical landmarks illustrated and dicuss the reason(s) why a faulty diagnosis could be made.
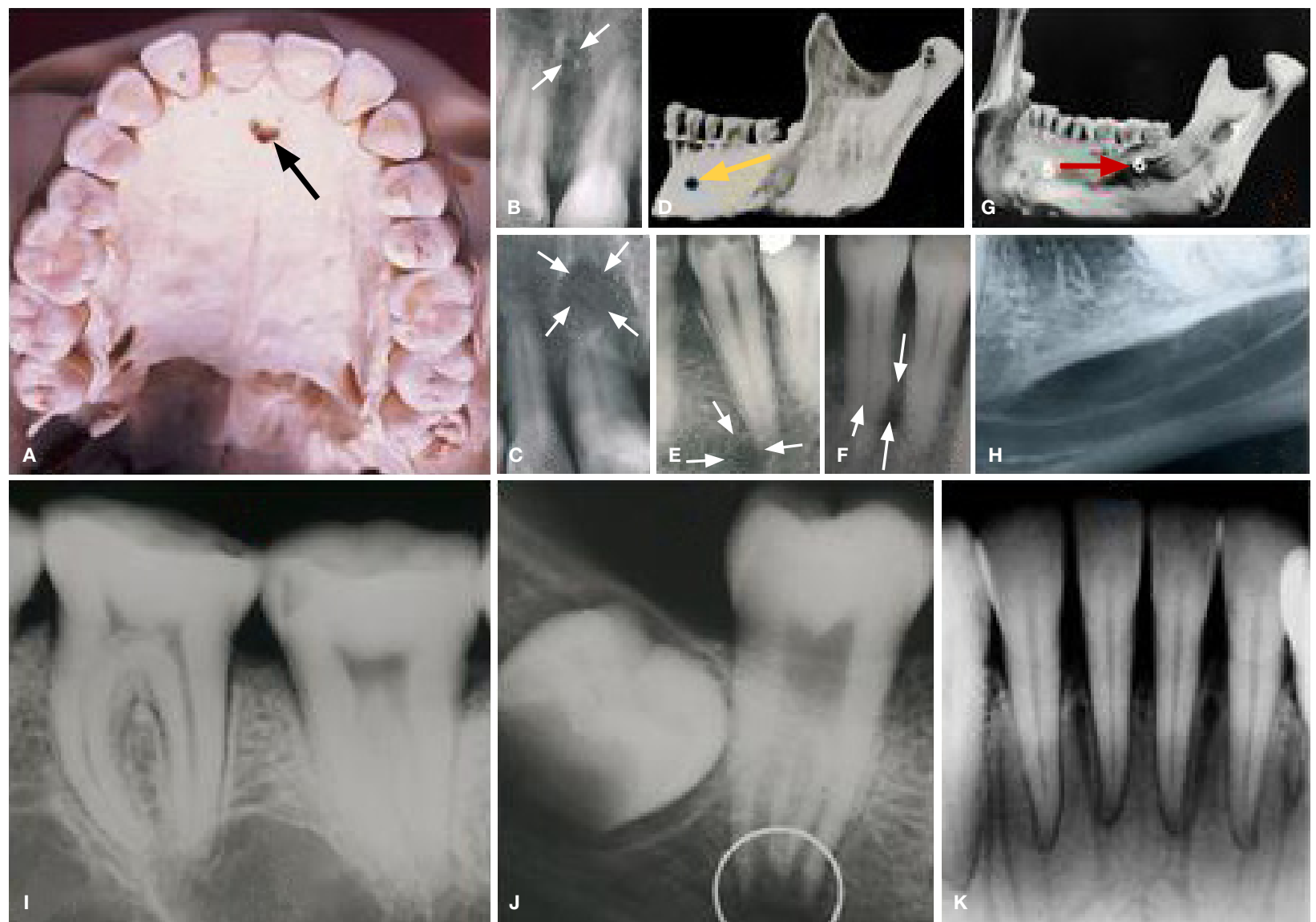

\section{INTERPRETATION}

Figures $A, B, C$, are radiographic images of the incisive foramen, which is an opening in the bone of the oral hard palate immediately behind the incisor teeth. In the incisive foramen the orifices of two lateral canals are visible; they are named the foramina of Stenson and additional canals are present in the middle line; they are termed the foramina of Scarpa (Fig. A \& B). A terminal branch of the maxillary nerve, the nasopalatine nerve, runs from the nasal cavity, through the incisive canal and supplies the tissues of the anterior part of the hard palate. Figure $\mathrm{C}$ shows a radiolucency at the apex of the 11 which could be diagnosed as an apical lesion instead of the incisive foramen due to a faulty angulation used during the $x$-ray examination. Figures D, E, show the normal position (yellow arrow) of the mental foramen through which the nerve and blood vessels emerge is seen as a round or oval radiolucency and but due to a faulty angulation during the taking of the $X$-ray one observe the abnormal position of the mental

Christoffel J Nortjé: $B C h D, P h D, A B O M R, D S c$. Faculty of Dentistry, University of the Western Cape.

ORCID Number: 0000-0002-9717-5514

Email: cnortje@uwc.ac.za foramen (Fig. F) suggesting the possible presence of a periodontal lesion. Figure $\mathrm{G}$ shows the mylohyoid ridge (red arrow) and submandibular fossa. According to the literature a prominent mylohyoid ridge and deep mandibular fossa may produce an image that may be mistaken for that of tumour or cyst (Fig. H). Fig. I shows two large radiolucency's suggestive of underlying pathology, however it is large marrow spaces presenting in the premolar and molar regions. Figure $\mathrm{J}$ is an example of a dentin papilla that is superimposed on the image of the mandibular canal producing a marked radiolucency suggestive of a periapical lesion which often occurs with the development of second and third molar teeth. Figure $\mathrm{K}$ shows multiple well demarcated vertical radiolucent lines suggestive of multiple fractures but the radiolucent lines lead directly to the apical foramina suggestive of interdental nutrient canals which are mostly observed in the edentulous regions. These canals contain blood vessels and nerves that supply the teeth and gingiva.

\section{References}

1. Stafne EC, Gibilisco JA. Oral Roentgenographic Diagnosis, $4^{\text {th }}$ Ed. Saunders. Philadelphia, London, Toronto. 1975; 1-7. 